\title{
Absence of intrinsic electric conductivity in single dsDNA molecules
}

\author{
H. Kleine ${ }^{\text {a }}$, R. Wilke ${ }^{\text {a }}$, Ch. Pelargus ${ }^{\text {a }}$, K. Rott ${ }^{\mathrm{b}}$, A. Pühler ${ }^{\mathrm{c}}$, \\ G. Reiss ${ }^{b}$, R. Ros ${ }^{\mathrm{a}}, \mathrm{D}$. Anselmetti ${ }^{\mathrm{a}, *}$ \\ ${ }^{a}$ Experimental Biophysics, Faculty of Physics, Bielefeld University, Universitätsstrasse 25, D-33615 Bielefeld, Germany \\ ${ }^{\mathrm{b}}$ Experimental Solid State Physics, Faculty of Physics, Bielefeld University, Universitätsstrasse 25, D-33615 Bielefeld, Germany \\ ${ }^{\mathrm{c}}$ Genetics, Faculty of Biology, Bielefeld University, Universitätsstrasse 25, 33615 Bielefeld, Germany
}

Received 15 December 2003; received in revised form 8 April 2004; accepted 16 April 2004

\begin{abstract}
The intrinsic dc conductivity of long, individual lambda phage dsDNA molecules has been investigated by ultrasensitive low current-voltage-spectroscopy (IV) under ambient conditions and controlled low humidity inert gas atmosphere on microfabricated metal-insulator-metal gap structures. We found a strong dependence of the measured conductivity on the apparent humidity, which we attribute to capillary condensation of water to the immobilized DNA molecules, giving rise to additional ionic currents. Additional IV-spectroscopy experiments under controlled argon atmosphere always revealed a significant drop in electrical conductivity to $4 \times 10^{-15} \mathrm{~A} \mathrm{~V}^{-1} \mu \mathrm{m}^{-1}$, indicating almost no considerable contribution of electrical long range charge transport.
\end{abstract}

Keywords: DNA conductivity; Current-voltage spectroscopy; Microstructures

\section{Introduction}

The investigation of intrinsic DNA conductivity has been highly debated over the last decade, especially since applications in molecular electronics or in new DNA-biosensor devices attracted the attention of many interdisciplinary working scientists (Keren et al., 2003). The pioneering work of Jacqueline Barton and coworkers who studied photoactivated charge transfer in small DNA fragments (Turro and Barton,

\footnotetext{
* Corresponding author. Tel.: +49-521-1065391; fax: +49-521-1062959.

E-mail address: dario.anselmetti@physik.uni-bielefeld.de (D. Anselmetti).
}

1998; Wan et al., 1999; Kelly and Barton, 1999; Núnez et al., 2001) and Bernd Giese and coworkers (Giese and Spichty, 2000; Giese et al., 2001; Meggers et al., 1998; Danith et al., 1999) who investigated the charge hopping of free cationic radicals along the distance of several base pairs of DNA fostered a very actual field. The investigation of long-range charge transport in DNA molecules were first reported by Fink and Schönenberger (1999) who found considerable charge transport by probing individual DNA molecules in vacuum by a low energy electron point source. In contrast to that, de Pablo et al. (2000) and Gómez-Navarro et al. (2002) reported no evidence of long range electrical charge transport in dsDNA of $\lambda$-phage type with sensitivity of $1 \mathrm{pA}$. In addition, 
Storm et. al. determined a lower bound limit for the resistance of DNA bundles DNA over gaps $>40 \mathrm{~nm}$ to $40 \mathrm{~T} \Omega$ (Storm et al., 2001).

Since to date, no group has paid subtle attention to environmental effects like humidity to the resistance of dsDNA molecules and the current-voltage spectroscopy experiments were mostly limited by the sensitivity of the experimental setup amplifier. We investigated the long range electrical transport through a limited number (1-10) of individual lambda DNA molecules under defined experimental conditions at a electric current sensitivity down to the attoampere level and $\mathrm{P} \Omega\left(10^{15} \Omega\right)$ resistance.

\section{Materials and methods}

\subsection{Metal-insulator-metal (MIM) microstructures}

Platinum electrodes on glass surfaces with gap sizes between 20 and $3000 \mathrm{~nm}$ were produced by standard lift off technology (Völklein and Zetterer, 2000; Wase, 2003). Briefly, photoresist (AR 535, Allresist, D) was spincoated with $4000 \mathrm{rpm}$ on clean glass slides (Menzel-Gläser, D) resulting in homogeneous film with a thickness of $1.2 \mu \mathrm{m}$ treated in a baking process $\left(95^{\circ} \mathrm{C}\right.$ and $\left.30 \mathrm{~min}\right)$. For contact lithography a flood exposure unit (Modell 8011, Oriel, USA) with a tantalum/gold mask was used. The masks are produced with a laser writer (DWL-66 Heidelberg instruments, D) in the same lift off process, as for the MIM structures. The coated glass slides were then developed in a developer bath (AR 300-26, Allresist) for $35 \mathrm{~s}$ and sputtered (Bal-Tec MED 20, Baltec, FL) with a $5-12 \mathrm{~nm}$ thick platinum layer. After $4 \mathrm{~min}$ in a remover bath (AR300-70, Allresist) the samples were rinsed with Millipore water and dryed under a stream of $\mathrm{N}_{2}$.

\subsection{Atomic force microscoy (AFM)}

To control number and alignment of DNA molecules AFM images were performed under ambient conditions in tapping mode with a Nanoscope IIIA Multimode (Veeco Instr., USA) using silicon cantilever with resonance frequencies of about $300 \mathrm{kHz}$ (NC, Nanosensors, D).

\subsection{Sample preparation}

In order to align single DNA molecules over the MIM structures a droplet of $0.3 \mu \mathrm{L}$ of $\lambda$-DNA (500 $\mu \mathrm{g} \mathrm{mL}^{-1}$, Promega, USA) was deposited on the gap. The meniscus forces during the drying process induces controlled orientation of the chain-like molecules often referred to as molecular combing (MC, Bensimon et al., 1994; Bensimon et al., 1995).

\subsection{I/V spectroscopy}

For the I/V spectroscopy measurements an ultrasensitive electrometer amplifier (6517A Keithley, USA) controlled by a home-made Labview program (National Instruments, USA) was used. For the experiments a sealed and electrically shielded stainless steel chamber for ambient, vacuum and argon conditions was designed.

\section{Results}

Double-stranded (ds) $\lambda$ DNA was deposited over MIM gap structures of 20-3000 nm (Fig. 1) by molecular combing. Upon controlling the DNA concentration in the solution and the surface chemistry, the number of DNA molecules witch the MIM gap junction can be controlled and tailored from molecular individuals, to molecular bundles, networks or complete DNA thin films. We mostly restricted our experiments to the investigations of defined molecular DNA individuals which were spread over the MIM gap microstucture as can be seen in the AFM images of Fig. 2. A typical current-voltage graph of such a structure with aligned DNA molecules is plotted in Fig. 3, where an electrical resistivity of $1.9 \mathrm{~T} \Omega$ at ambient conditions can be measured. In order to check for parasitic humidity effects which might arise from capillary condensation of water to the negatively charged backbone of dsDNA (Otsuka et al., 2002), we investigated the same molecular IV-curve by placing the molecular MIM device in a humidity free argon inert gas atmosphere. After a typical time of 5 min which we allowed the system to equilibrate we could always detect a significant drop in electric conductivity and therefore an almost complete absence of long range dc 

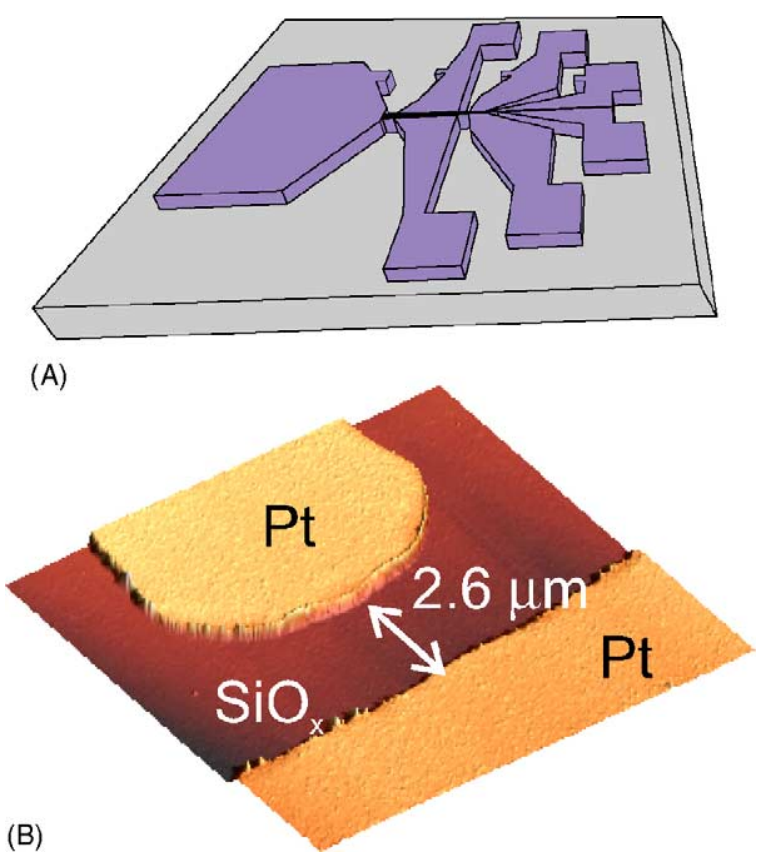

Fig. 1. Microfabricated MIM-gap structures. (A) Schematic layout of the electrodes. (B) AFM image of two electrodes forming a $2.6 \mu \mathrm{m}$ gap.

electric charge transport. Since the measured electric currents were in the femtoampere regime we replotted the experimental data with an adjusted $y$-scale for better visibility (Fig. 4).

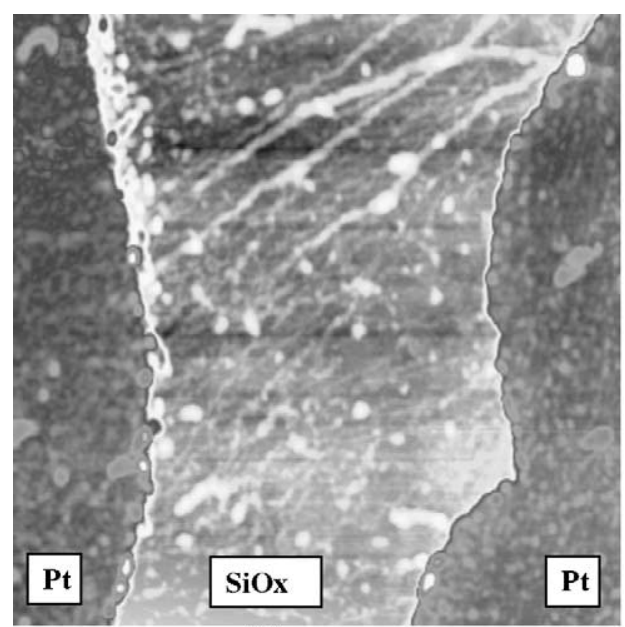

(A)

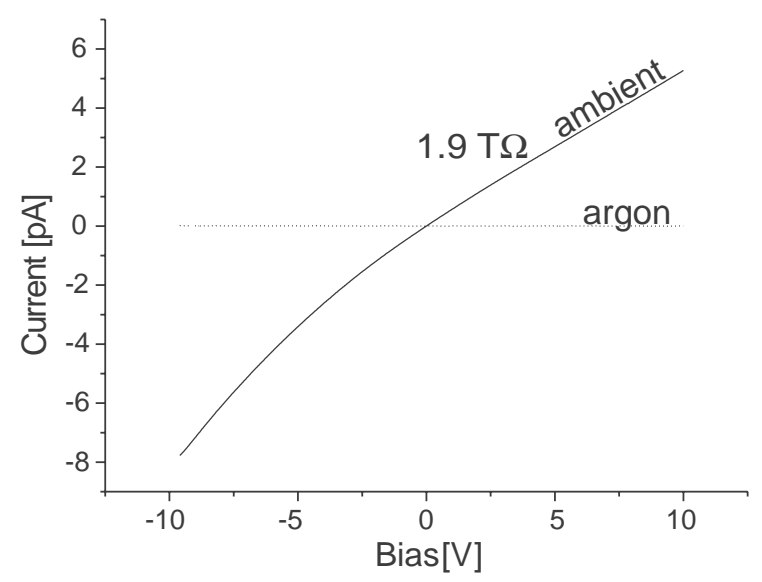

Fig. 3. Current-voltage graph of a $550 \mathrm{~nm}$ gap structure covered with dsDNA. Under ambient conditions and under controlled inert gas (argon) atmosphere.

\section{Discussion}

In this ultrasensitive current regime the complete measurement set up is highly sensitive to electrical (de)charging of the shielding cables, connectors and even the gap structures. In order to account for these effects we kept all external parameters (cable length, temperature) constant and analyzed the IV data within the model of an electric device in which a resistor is paralleled by an condenser (see inlet Fig. 4). By

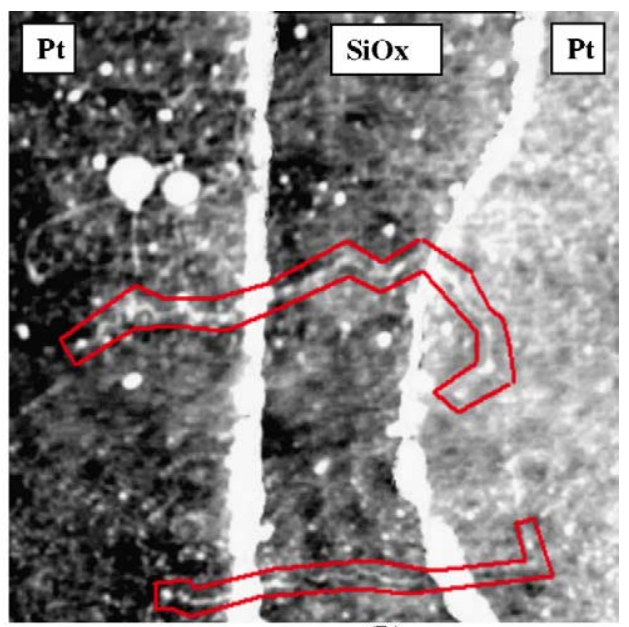

(B)

Fig. 2. AFM images of individual dsDNA molecules immobilized on MIM gap structure by molecular combing. (A) Scan size $1.2 \mu m$ and (B) $1.5 \mu \mathrm{m}$. 


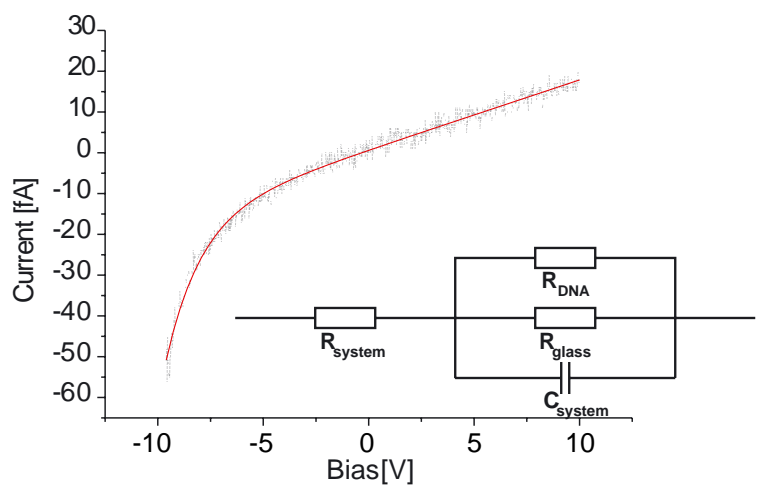

Fig. 4. Ultrasensitive IV spectroscopy curve of dsDNA under argon atmosphere (grey). The black line indicates the fit of these data with a model based on the block diagram (inlet).

applying a straightforward classical theory to compensate for the (de)charging effects of the cables we found the following simplified equation:

$$
\begin{aligned}
I(u)= & I_{\mathrm{c}}+\frac{U(t)}{\left(R_{\mathrm{DNA}} R_{\text {glass }} /\left(R_{\mathrm{DNA}}+R_{\text {glass }}\right)\right)+R_{\text {system }}} \\
& +I_{0} \exp \left(-\frac{U(t)}{\lambda}\right)
\end{aligned}
$$

with the resistors $R_{\mathrm{DNA}}, R_{\text {glass }}$ and $R_{\text {system }}$ shown in Fig. 4 and $I_{\mathrm{c}}, \lambda$, and $I_{0}$ denote device dependent constants, respectively. Fits of IV data of gaps with and without DNA result in the same intrinsic conductivity $\left(I / R_{\mathrm{DNA}}\right)$ within the errors of measurement of about $4 \times 10^{-15} \mathrm{~A} \mathrm{~V}^{-1} \mu \mathrm{m}^{-1}$. This remarkably low value, which corresponds to a lower bound of a $\mathrm{P} \Omega$ resistance, indicating an almost complete absence of dc electric charge transport in DNA and is very close to the conductivity values of MIM gap structures without DNA molecules. This negative results was verified several dozen times with a limited number of DNA molecules under controlled inert gas atmosphere without having any evidence of DNA conductivity at all, indicating an absence of dc-conductivity in single molecules.

Since we measured only a significant conductivity in DNA under ambient conditions at laboratory humidity values of 30-60\%, we conclude that the earlier reported DNA conductivities can be explained either by ionic transport through capillary condensation in the vicinity of the deposited DNA molecules (Hwang et al., 2002; Kasumov et al., 2001; Lee et al., 2002;
Hwang et al., 2002; Otsuka et al., 2002; Cai et al., 2000; Yoo et al., 2001) or other transport phenomena like through a carbon mantle from the cracked carbohydrate residual in vacuum by electrons and deposition on the DNA strand (de Pablo et al., 2000; Gómez-Navarro et al., 2002; Fink and Schönenberger, 1999).

In summary, we found that the intrinsic electrical properties of dsDNA for long range charge transport of $\mathrm{P} \Omega \mu \mathrm{m}$ is far below of any technological use. A possible way to improve the electrical conductivity might be additional doping of dsDNA or molecular engineering of hybrid systems (Keren et al., 2003; Brown et al., 1998) which can be used in future devices for molecular electronics or biosensor applications.

\section{Acknowledgements}

This research project has been funded and supported by the collaborative research project SFB 613 from the Deutsche Forschungsgemeinschaft. We thank J. Schotter for technical support with the laser writer.

\section{References}

Bensimon, A., Simon, A., Chiffaudel, A., Croquette, V., Heslot, F., Bensimon, D., 1994. Alignment and sensitive detection of DNA by a moving interface. Science 265, 2096.

Bensimon, A., Simon, A.J., Chiffaudel, A., Croquette, F., Bensimon, D., 1995. Stretching DNA with a receding meniscus: experiments and models. Phys. Rev. Lett. 75, 4754.

Brown, E., Eichen, Y., Sivan, U., Ben-Yoseph, G., 1998. DNAtemplated assembls and electrode attachment of a conducting silver wire. Nature 391, 775.

Cai, L., Tabata, H., Kawai, T., 2000. Selfasembled DNA networks and their electrical conductivity. Appl. Phys. Lett. 77, 3105.

Danith, L., Laurie, S., Schuster, G.B., 1999. Mechanism of charge transport in DNA: internally-linked anthraquinone conjugates support phonon-assisted polaron hopping. J. Am. Chem. Soc. 121, 9400.

de Pablo, P.J., Moreno-Herrero, F., Colchero, J., Gómez-Herrero, J., Herrero, P., Baró, A.M., Ordejón, P., Soler, J.M., Artacho, E., 2000. Absence of dc-conductivity in 1-DNA. Phys. Rev. Lett. 85, 4992.

Fink, H.-W., Schönenberger, C., 1999. Electrical conduction through DNA molecules. Nature 398, 407.

Giese, B., Spichty, M., 2000. Long distance charge transport through DNA: quantification and extension of the hopping model. Chem. Phys. Chem. 1, 195. 
Giese, B., Spichty, M., Wessely, S., 2001. Long-distance charge transport through DNA: An extended hopping model. Pure Appl. Chem. 73 (3), 449.

Gómez-Navarro, C., Moreno-Herrero, F., de Pablo, P.J., Colchero, J., Gómez-Herrero, J., Baró, A.M., 2002. Contactless experiments on individual DNA molecules show no evidence for molecular wire behavior. PNAS 99 (13), 8484.

Hwang, J.S., Lee, G.S., Kong, K.J., Ahn, D.J., Hwang, S.W., Ahn, D., 2002. Electrical transport $60 \mathrm{bp}$ of $\operatorname{poly}(\mathrm{G})-\operatorname{poly}(\mathrm{C})$ DNA molecules. Appl. Phys. Lett. 81, 1134.

Hwang, J.S., Lee, G.S., Kong, K.J., Ahn, D.J., Hwang, S.W., Ahn, D., 2002. Electrical transport through poly(G)-poly(C) DNA molecules. Microelectron. Eng. 63, 161.

Kasumov, A.Yu., Kociak, M., Guéron, S., Reulet, B., Volkov, V.T., Klinow, D.V., Bouchiat, H., 2001. Proximity-induced superconductivity in DNA. Science 291, 280.

Kelly, S.O., Barton, J.K., 1999. Electron transfer between bases in double helical DNA. Science 283, 375.

Keren, K., Berman, R.S., Buchstab, E., Sivan, U., Braun, E., 2003. DNA-templated carbon nanotube field-effect transistor. Science 302,1380 .

Lee, H.-Y., Tanaka, H., Otsuka, Y., Yoo, K.-H., Lee, J.-O., Kawai, T., 2002. Control of electrical conduction in DNA using oxygen hole doping. Appl. Phys. Lett. 80, 1670.
Meggers, E., Michel-Beerle, M.E., Griese, B., 1998. Sequence dependent long range hole transport in DNA. J. Am. Chem. Soc. $120,12950$.

Núnez, M.E., Holmquist, G.P., Barton, J.K., 2001. Evidence for DNA charge transport in the nucleus. Biochemistry 40 (42), 12465.

Otsuka, Y., Lee, H.-Y., Gu, J.-H., Lee, J.-O., Yoo, K.-H., Tanaka, H., Tabata, H., Kawai, T., 2002. Influence of humidity on the electrical conductivity of synthesized DNA film on nanogap electrode. Jpn. J. Appl. Phys. 41, 891.

Turro, N.J., Barton, J.K., 1998. Paradigms, supermolecules, electron transfer and chemistry at a distance. What's the problem? The science or the paradigm? JBIC 3, 201.

Völklein, F., Zetterer, T., 2000. Einführung in die Mikrosysemtechnik. Vieweg-Verlag, Braunschweig/Wiesbaden.

Wan, C., Fiebig, T., Kelly, S.O., Treadway, C.R., Barton, J.K., Zewail, H., 1999. Femtosecond dynamics of DNA-mediated electron transfer. Proc. Natl. Acad. Sci. U.S.A. 96, 6014.

Wase, R. (Ed.), 2003. Nanoelectronics and information technology. Wiley-VHC, Weinheim.

Yoo, K.-H., Ha, D.H., Lee, J.-O., Park, J.W., Kim, J., Kim, J.J., Lee, H.-Y., Kawai, T., Choi, H.Y., 2001. Electrical conduction through poly(dA)-poly(dT) and poly(dG)-poly(dC) DNA molcecules. Phys. Rev. Lett. 87, 8102. 